\title{
The Role of Aldosterone Blockade in Patients with Heart Failure
}

Bertram Pitt, MD

The University of Michigan School of Medicine, Ann Arbor, MI 48170, USA

\begin{abstract}
The mechanisms associated with aldosterone production both systemically and locally as well as the effects of aldosterone blockade on the pathophysiology of heart failure (HF) have been extensively reviewed in this series and elsewhere. This article will review the clinical evidence supporting the use of aldosterone blocking agents (AB)in patients with HF and speculate on some potential future uses.
\end{abstract}

\section{Results of Major Clinical Trials of $A B$ in Patients with HF}

The randomized Aldosterone evaluation study (RALES) [1] was the first study to show the benefit of $\mathrm{AB}$ on mortality as well as hospitalization for HF. In Rales over 1600 patients with a history of severe HF (NYHA class IV within the 6 months prior to entry into the study) who were in NYHA class III-IV and maintained on standard therapy including an angiotensin converting enzyme-inhibitor (ACE-I), beta blocker (BB), diuretic, and digoxin were randomized to the $\mathrm{AB}$ spironolactone $25-50 \mathrm{mg} /$ day or placebo with the primary endpoint of total mortality. Patients entered into the study had to have evidence of systolic left ventricular dysfunction (SLVD) with a left ventricular ejection fraction (LVEF) $\leq 35 \%$. The study was stopped prematurely at a mean followup of 2 years when it was found that patients randomized to the $\mathrm{AB}$ strategy had a significant $30 \%$ reduction in total mortality as well as a $35 \%$ reduction in the incidence of hospitalization for HF. The reduction in total mortality was due both to a reduction in death due to progressive $\mathrm{HF}$ and sudden cardiac death. These benefits were relatively uniform across a number of predetermined subgroups including age, gender, etiology of HF, and whether or not the patient was on an ACE-I, BB, diuretic, or digoxin. Initially there was some reluctance to apply the results of this study to clinical practice since only $10-11 \%$ of patients were on a $\mathrm{BB}$, in view of the fact that this study was initiated before the results of the large scale BB event trials in patients with $\mathrm{HF}$ were known. However, the point estimate for a reduction in mortality in those patients on a BB was even greater than in those not on a BB. More importantly the results of the Eplerenone Heart Failure and Survival study (EPHESUS) [2] of the AB eplerenone $25-50 \mathrm{mg} /$ day in which $75 \%$ of patients were on a BB and $86-87 \%$ on an ACE-I or angiotensin receptor blocking agent (ARB) showed the effectiveness of an $\mathrm{AB}$ in reducing mortality in patients with HF or SLVD post myocardial infarction (MI) on both an ACE-I or ARB and a BB. On the basis of Rales $\mathrm{AB}$ is now recommended for patients with severe $\mathrm{HF}$ in both ACC/AHA and European guidelines and is finding increasing application.

$\mathrm{AB}$ have also been shown to be beneficial in patients with HF and SLVD early post MI. EPHESUS [2] randomized over 6600 patients between day 3-14 following an acute MI with a LVEF $\leq 40 \%$ and clinical signs of $\mathrm{HF}$, unless they had diabetes mellitus in which case only a LVEF $\leq 40 \%$ was required, to usual care or eplerenone $25 \mathrm{mg}$ /day uptitrated to $50 \mathrm{mg} /$ day at 1 month if there was no evidence of hyperkalemia (potassium $>5.0 \mathrm{meq} / \mathrm{l}$ ). Over $85 \%$ of patients were on an ACE-I or an ARB and $75 \%$ on a BB. Slightly less than half the patients were on statin and had undergone coronary reperfusion. The mean time from onset of infarction to randomization was 7.3 days and the mean LVEF 33\%. However, the actual LVEF at the time of randomization was likely considerably greater than $33 \%$ since the LVEF was determined at any time from the onset of MI to randomization by which time many patients had recovered from ventricular stunning, hibernation, and or had undergone coronary reperfusion.

At a mean follow up of 16 months patients randomized to eplerenone had a significant $15 \%$ reduction in total mortality and a $13 \%$ reduction in the coprimary endpoint of CVmortality/CV hospitalization. The major cause of cardiovascular death in Ephesus was sudden cardiac death which was significantly reduced by $21 \%$. In a retrospective analysis of those patients with a LVEF $\leq 30 \%$ at baseline there was a $33 \%$ reduction in sudden cardiac death. Of importance was the finding that at 30 days post randomization (a mean of 37 days post $\mathrm{MI}$ ) there was a significant $31 \%$ reduction in total mortality. The finding of a significant 
reduction in total mortality in these patients has important implications since patients with $\mathrm{HF}$ and or SLVD post MI have a greatly increased risk of mortality, mainly due to sudden cardiac death, within the first 30 days post MI despite treatment with an ACE-I and a BB. Early implantation of an automatic implantable defibrillator (AICD) would appear to be a useful strategy in these high risk individuals. However the DYNAMIT [3] study which randomized patients with an acute MI between day 6 to 40 post MI with a LVEF $\leq 35 \%$ found that those patients randomized to the AICD strategy had an increase in total mortality at 1 year due to an excess in non-sudden deaths. The Madit 2 trial did show a significant reduction in total mortality in patients with a LVEF $\leq 30 \%$ randomized to a usual care or AICD strategy $>30$ days post MI [4]. However, benefit in that study was nor evident until almost 1 year post randomization. Thus, it has been suggested that eplerenone could be an important bridging strategy which could prevent ventricular remodeling and therefore decrease the number of patients with an LVEF $\leq 30 \%$ at 30 days post MI and in those patients with a persistent LVEF $\leq 30 \%$ reduce early mortality such that they would be alive to benefit from 0the long term beneficial effects of AICD implantation. Although in EPHESUS [2] eplerenone was given at a mean of 7.3 days post MI a subsequent study by Hyashi et al. [5] in patients with their first anterior MI the $\mathrm{AB}$ canreonate was administered iv on day 1 post MI after primary percutaneous coronary revascularization and followed by oral spironolactone for 1 month in a randomized protocol. In that study $\mathrm{AB}$ was shown to significantly prevent ventricular remodeling and collagen formation at 1 month. Although there has not been a large scale event trial of an $\mathrm{AB}$ administered on day 1 post $\mathrm{MI}$ it would seem most efficacious given the results of EPHESUS [2] and that of Hyashi et al. [5] to administer an $\mathrm{AB}$ as soon as possible post $\mathrm{MI}$ in patients with evidence of HF and or SLVD to impact the relatively high mortality in these individuals. On the basis of the EPHESUS [2] study administration of an $A B$ is now a class I indication post $M I$ in patients with HF and SLVD post MI. It is however likely that $\mathrm{AB}$ will also be effective in reducing cardiovascular events in patients without evidence of $\mathrm{HF}$ or an LVEF $\leq 40 \%$ post MI, in that the mean LVEF at baseline in the study of Hyashi et al. [5] was approxamitely $47 \%$ and patients were not required to have evidence of HF.

Both in RALES [1] and EPHESUS [2] there was an increase in the incidence of serious hyperkalemia $(K \geq 6.0 \mathrm{meq} / \mathrm{l})$. However the risk was relatively small and no patient in RALES [1] or EPHESUS [2] randomized to an $\mathrm{AB}$ had a death attributable to hyperkalemia.

\section{Patient Monitoring of Serum Potassium in Patients with HF Treated with an AB}

On the basis of the experience in RALES [1] and EPHESUS [2] one should obtain a serum potassium and calculate creatinine clearance before beginning an $\mathrm{AB}$. Patients with a serum $K>$ $5.0 \mathrm{meq} / \mathrm{l}$ and or those with severe renal dysfunction (Creatinine clearance $\leq 30 \mathrm{ml} / \mathrm{min}$ ) in general should not receive an $\mathrm{AB}$. After instituting an $\mathrm{AB}$ serum potassium should be determined at 1 week, 1 month, and then every 3-6 months thereafter. In patients with moderate renal dysfunction and a creatinine clearance $<60->30 \mathrm{ml} / \mathrm{min}$ it might be prudent to monitor serum potassium weekly for the first month and then monthly thereafter for the first several months. This sequence should be repeated every time the dose of the $A B$ is increased, when there is a change in fluid balance such as vomiting or diarrhea, and or when there is a change in medication which could effect potassium excretion such as a non steroidal antiinflammatory agent.

If at any time serum potassium is $\geq 5.5 \mathrm{meq} / \mathrm{l}$ the dose of the $A B$ should be reduced by half and serum potassium monitored weekly until serum potassium is $<5.5 \mathrm{meq} / \mathrm{l}$. If at any time serum potassium is $\geq 6.0 \mathrm{meq} / \mathrm{l}$ on a non hemolyzed sample a careful review of concomitant medication should be undertaken and if there is no precipitating factor such as the use of a non steroidal anti-inflammatory agent or Cox-2 inhibitor the $A B$ should be discontinued and not restarted until the serum potassium is $<5.0 \mathrm{meq} / \mathrm{l}$ and or any precipitating factor is eliminated. If spironolactone is chosen the initial dose should be $25 \mathrm{mg} /$ day as in RALES [1]. However, in elderly patients ( $>75$ years),those with diabetes mellitus and evidence of microalbuminuria, and or those with compromised renal function it might be prudent to begin dosing with $25 \mathrm{mg}$ every other day. If after 1 month there is no increase in serum potassium $>5.0 \mathrm{meq} / 1$ the dose of spironolactone could be increased to $25 \mathrm{mg}$ daily. In patients with evidence of progressive $\mathrm{HF}$ and or in those patients with diuretic resistance higher doses of spironolactone up to $200 \mathrm{mg}$ /day have been shown to be effective in causing diuresis. Once diuresis is achieved it might however be prudent to reduce the dose to $25-50 \mathrm{mg} /$ day. If eplerenone is chosen the starting dose is $25 \mathrm{mg} /$ day, which is relatively lower than $25 \mathrm{mg}$ of spironolactone. If after 1 month there is no evidence of hyperkalemia the dose should be increased to $50 \mathrm{mg} /$ day. In contrast to spironolactone there is however no significant experience with higher doses of eplerenone in patients with diuretic resistance, although $50 \mathrm{mg}$ twice daily is approved for use in patients with hypertension. 


\section{Which AB Should One Use in a Patient with HF?}

Both spironolactone and eplerenone are effective inhibitors of the minerallocorticoid receptor (MR). While there are no definitive comparative trials in patients with HF there is data to reach at least a provisional answer in regard to the choice of one or the other in particular circumstances. A major difference between spironolactone and eplerenone relates to their specificity for the MR. Spironolactone is less specific for the MR than eplerenone and down regulates androgen receptors while upregulating progesterone receptors [6]. Spironolactones use is therefore associated with gynechomastia, breast pain and impotence in males as well as menstrual irregularities and libido changes in premenstrual females. In EPHESUS [2] there was no evidence of an increase in any of these sexually related side effects known to be associated with the use of spironolactone in over 3300 patients followed on eplerenone for a mean of 16 months, attesting to its relative selectivity for the MR. Spironolactone also has a relatively long half life of over 24 hours due to its metabolite potassium canreonate in contrast to eplerenone which has a relatively shorter half life [7]. This difference in half life could impact the relative incidence of serious hyperkalemia. Although there have been no adequately powered comparative studies one would predict that the incidence of serious hyperkalemia would be less with eplerenone than sprionolactone. Spironolactone also is known to increase serum digoxin levels while eplerenone does not. Another difference is cost. Spironolactone is generic and cost less than $\$ 1$ per day in comparison to eplerenone which was recently introduced into clinical practice and costs over $\$ 3$ per day. Thus in males, premenstrual females, and in those with compromised renal function one might therefore favor the use of eplerenone. In post menopausal females with normal renal function spironolactone might be preferred due to cost. If cost is the overrding issue and the patient can not afford eplerenone I would recommend spironolactone in males, premenstrual females and in those with compromised renal function rather than doing without an $\mathrm{AB}$. It should however be emphasized that these recommendations are provisional and await more definitive information from direct comparative trials.

\section{Which Patients with HF and SLVD Should be Treated with an AB?}

Patients with severe or progressive HF and SLVD both due to ischemic and non ischemic heart disease should be treated with an $\mathrm{AB}$ in addition to an ACE-I, BB, diuretic +/- digoxin based upon the results of RALES [1]. Similarly, patients with $\mathrm{HF}$ and SLVD post MI should be given an $\mathrm{AB}$ as early as possible post MI based upon the results of EPHESUS [2] and the AB continued indefinitely in those with persistent SLVD and or evidence of $\mathrm{HF}$.

The situation in patients with mild heart failure and SLVD is less clear as there have as yet been no definitive trials addressing mortality/hospitalization for $\mathrm{HF}$ in this situation. In these patients one might chose an ARB to add to an ACE-I and a BB. The use of an ARB could be justified on the basis of the Charm-added trail [8] in which the addition of candesartan $32 \mathrm{mg} /$ day to standard therapy including an ACE-I and BB significantly reduced CV mortality/hospitalization for HF. However in Valheft [9], in a similar population, valsartan $160 \mathrm{mg}$ twice daily did not have a beneficial effect on mortality in patients treated with both an ACE-I and a BB. There was however a significant reduction in hospitalization for $\mathrm{HF}$ with valsartan, but even this effect was lost in patients who achieved target doses of their ACE-I.

In patients with $\mathrm{HF}$ and or SLVD early post MI valsartan was shown to be equivalent in effectiveness to the ACE-I captopril [10]. However there was no benefit of adding valsartan to captopril.Thus the efficacy of adding an ARB to an ACE-I and a BB both in patients with chronic $\mathrm{HF}$ due to SLVD and in patients with $\mathrm{HF}$ and or SLVD early post MI is uncertain. While there is no large scale randomized study evaluating the use of an $A B$ in patients with mild $\mathrm{HF}$ due to SLVD there have been several small randomized studies evaluating surrogate endpoints [11-14]. These studies suggest that the addition of an $\mathrm{AB}$ to standard therapy including an ACE-I and a BB improves these endpoints including ventricular remodeling, collagen formation, endothelial function, heart rate variability, baroreceptor function, ventricular ectopic activity, and exercise performance. While there may be some benefit of adding an ARB to an ACE-I in preventing the production of ATII and or in preventing stimulation of the AT1 receptor there is an equal or better rationale for adding an AB. Although ATII is a potent stimulus for the production of ATII other stimuli such as potassium are also important. For example, in the angiotensinogen knock out mouse, in which ATII is not present, aldosterone production from the adrenal gland can be obtained by modulating serum sodium [15]. Further, while both ATII and aldosterone share common signaling pathways through the epidermal derived growth factor receptor (EDGFR) they also have independent signaling pathways, suggesting that optimum benefit will be obtained by blocking or inhibiting both ATII and aldosterone [16]. Experimental studies 
have shown a significantly better effect on ventricular remodeling post MI when an ACE-I is combined with an $\mathrm{AB}$ in comparison to either alone [17]. Further prospective randomized studies in patients with chronic HF due to SLVD comparing the strategy of adding an $\mathrm{ARB}$, an $\mathrm{AB}$, or both to an ACE-I and a BB will however be required before any final conclusion can be reached.

\section{Future Application of $A B$ in Patients with $\mathbf{H F}$}

The use of an $\mathrm{AB}$ in patients with asymptomatic SLVD has not been studied. ACE-I and BB are effective in reducing $\mathrm{CV}$ risk in these patients. It is possible, although as yet unproven, that the addition of an $A B$ in these patients who have systolic ventricular dysfunction and activation of the RAAS would provide further benefit.

The role of $\mathrm{AB}$ in patients with $\mathrm{HF}$ associated with preserved ventricular function is also uncertain. Increasing data both in animals and man does however suggest a beneficial effect of an $\mathrm{AB}$ in this situation. $\mathrm{AB}$ has been shown to regress ventricular hypertrophy, myocardial collagen formation, vascular stiffness, and to improve endothelial function [18-21]. $\mathrm{AB}$ has also been shown to improve echocardiograpic indicies of diastolic function in patients with diastolic $\mathrm{HF}$ [20]. The hypothesis that an AB added to standard therapy will improve clinical outcome (CV mortality/hospitalization for $\mathrm{HF}$ ) in patients with $\mathrm{HF}$ associated with preserved ventricular function is being tested by the NHLBI in a large scale prospective randomized trial.

There is also reason to postulate that $\mathrm{AB}$ will be beneficial in other forms of $\mathrm{HF}$ such as $\mathrm{HF}$ due to valvular heart disease, myocarditis, and drug induced cardiomyopathy. $\mathrm{AB}$ may also have an important role in the prevention of HF by preventing target organ damage associated with essential hypertension and or diabetes mellitus,as well as preventing the progression of atherosclerosis. Experimental studies have shown that AB decreases the production of reactive oxygen spieces (ROS), possibly related to an improvement in antioxidant reserve through an increase in the enzyme glucose 6 phosphatedehydrogenase (G6PD) with a consequent increase in glutathione [22]. $\mathrm{AB}$ has also been shown to improve lipid induced endothelial dysfunction [23], macrophage oxidized LDL-cholesterol [24], and experimental atherosclerosis in the apo-e knock out mouse [25]. Clearly although $\mathrm{AB}$ has achieved an important role in the therapy of patients with chronic $\mathrm{HF}$ and SLVD as well as in patients with HF and SLVD post MI there remains much to be learned about the clinical application of $\mathrm{AB}$ to patients with $\mathrm{HF}$.

\section{References}

1. Pitt, B, Zannad F, Remme WJ, Cody R, Castaigne A, Perez A, et al. The effect of spironolactone on morbidity and mortality in patients with severe heart failure. $N \mathrm{Engl} J \mathrm{Med}$ 1999;341:709-717.

2. Pitt, B, Remme W, Zannad F, Neaton J, Martinez F, Roniker B, Bittman R, Hurley S, Kleiman J, Gatlin M, of the Eplerenone Post-Acute Myocardial Infarction Heart Failure Efficacy and Survival study Investigators. Eplerenone, a selective aldosterone blocker, in patients with left ventricular dysfunction after myocardial infarction. $N$ Eng $J$ Med 2003;348(14):1309-1321.

3. Hohnloser SH, Heinz Kuck K, Dorian P, Roberts RS, Tech M, Hampton JR, Hatala R, Fain E, Gent M. Connolly. Prophylacticuse of an implantable cardioverterdefibrillator after acute myocardial infarction. SJ NEJM 2004;351:2481-2488.

4. Moss AJ, Zareba W, Hall WJ, Klein H, Wlber DJ, Cannom DS, et al. Prophylactic implantation of a defibrillator in patients with myocardial infarction and reduced ejection fraction. N Engl J Med 2002;346:877-883.

5. Hayashi M, Tsutamoto T, Wada A, et al. Immediate administration of mineralocorticoid receptor antagonist spironolactone prevents post-infarct left ventricular remodeling associated with suppression of a marker of myocardial collagen synthesis in patients with first anterior acute myocardial infarction. Circulation 2003;107:2259-2265.

6. de Gasparo M, Joss U, Ramjoue HP. Three new epoxyspironolactone derivatives: Characterization in vivo and in vitro. J. Pharmacol Exp Ther 1987;240:650-656.

7. Ravis WR, Reid S, Roniker B, et al. Pharmacokinetics of eplerenone after single and multiple dosing in subjects with and without renal impairment. Clin Pharmacol There 2004;75:P37.

8. Pfeiffer MA, McMurray JJ, Valazques EJ, Rouleau JL, Kober L, Maggioni AP, et al. Valsartan, captopril, or both in myocardial infarction complicated by heart failure, left ventricular dysfunction, or both. $N$ Eng $J$ Med 2003;349:1893-1906.

9. Cohn JN, Tognoni G. A randomized trial of the angiotensinreceptor blocker valsartan in chronic heart failure. $N$ Engl J Med 2001:345:1667-1675.

10. Pfeffer MA, McMurray JJ, Velazquez EJ, Rouleau JL, Kober L, Maggioni AP, Solomon SD, Swedberg K, Van de Werf F, White H, Leimberger JD, Henis Marc, Edwards S, Zelenkofske S, Sellers MA, Califf RM. For the Valsartan in Acute Myocardial Infarction Trial Investigators. Valsartan, captopril, or both in myocardial infarction complicated by heart failure, left ventricular dysfunction or both. $N$ Eng J Med 2003;349:1893-1906.

11. Tsutamoto T, Wada A, Maeda K, Mabuchi N, Hayashi M, Tsutsui T, Ohnishi M, Sawaki, M, Fujii M, Matsumoto T, Matsui T, Kinoshita M. Effect of spironolactone on plasma brain natriuretic peptide and left ventricular remodeling in patients with congestive heart failure. J Am Coll Cardiol 2001;37:1228-1233.

12. Cicoira M, Zanolla L, Rossi A, Golia G, Franeschini L, Brighetti G, Marino P, Zardini P. Long-term, dose dependent effects of spironolactone on left ventricular function and exercise tolerance in patients with chronic heart failure. J Am Coll Cardiol 2002;40:304-310.

13. MacFadyen RJ, Barr CS, Struthers AD, Aldosterone blockade reduces vascular collagen turnover, improves heart 
rate variability and reduces early morning rise in heart rate in heart failure patients. Cadiovasc Res 1997;35:3034.

14. Meyer B, Huelsmann M, Strecker K, Neunteufl T, Pacher $\mathrm{R}$, Berger R. Flow-mediated vasodilation predicts outcome in patients with chronic heart failure. JACC 2004;43:198A.

15. Okubo S, Niimura F, Nishimura H, Takemoto F, Fogo A, Matsusaka T, Ichikawa I. Angiotensin-independent mechanism for aldosterone synthesis during chronic extracellular fluid volume depletion. Journal of Clinical Investigation 1997:855-860.

16. Mazak I, Fiebler A, Dominik N, et al. Aldosterone potentiates Angiotensin II-induced signaling in vascular smooth muscle cells. Circulation 2004;109:2792-2800.

17. Fraccarollo D, Galuppo P, Hildemann S., Christ M, Ertl G, Baursachs J. Additive improvement of lefr ventricular remodeling and neurohormonal activation by aldosterone receptor blockade with eplerenone and ACE inhibition in rats witn myocardial infarction. JACC 2003;42:1666-1673.

18. Susic D, Varagic J, Ahn I, Matavelli L, Frolich ED, Eplerenone improves coronary hemodynamics and reduces cardiac fibrosis in aging spontaneously hypertensive rats. JACC 2004;43:482A.

19. LaColley P, Labat C, Pujol A, Delcayre C, Benetos A, Safar M. Increased carotid wall elastic modulus and fibronectin in aldosterone-salt-treated rats effects of eplerenone. Circulation 2002;106:2848-2853.

20. Mottram P, Haluska B, Leano R, Cowley D, Stowasser M, Marwick T. Aldosterone antagonism in hypertensive HD. Circulation 2004;110:558-565.

21. Pitt B, Reichek N, Willenbrock R, et al. Effect of eplerenone, enalapril, and eplerenone/enalapril in patients with essential hypertension and left ventricular hypertrophy study. Circulation 2003;108:1831-1838.

22. Leopold JA, Scribner AE, Dam A, Lioa R. Aldosterone impairs vascular reactivity by decreasing glucose Gphosphate dehydrogenase activity. Circ 2004;110:III154.

23. Rajagopalan S, Duquaine D, King S, Pitt B, Patel P, Mineralocorticoid receptor antagonism in experimental atherosclerosis. Circulation 2002;105:2212-2216.

24. Keidar S, Kaplan M, Pavlotsky E, et al. Aldosterone administration to mice stimulates macrophage NADPH oxidase and increases atherosclerosis development: A possible role for angiotensin-converting enzymes and the receptors for angiotensin II and aldosterone. Circulation 2004;109:2213-2220.

25. Zhou Y, Chen $\mathrm{R}, \mathrm{Hu}$, et al. Angiotensin II and aldosterone are both required to stimulate atherosclerosis in apo-E deficient mice. Hyp , 2003;42:403A Abstract 83. 